\title{
La formación profesional en la construcción de trayectorias laborales. Estudio sobre sectores productivos en Argentina ${ }^{1}$
}

\author{
Vocational Training in the Construction of Work Trajectories. \\ Survey on Productive Sectors in Argentina
}

\author{
Marcelo Fabian Delfini, María Ana Drolas y Juan Sebastian Montes Cató2
}

\begin{abstract}
Resumen
El artículo se ubica en el campo problemático entre educación y trabajo buscando caracterizar de manera específica la formación profesional -FP_. Para ello interesa analizar el lugar que ocupa este tipo de formación en las trayectorias laborales de los trabajadores. El texto parte de un estudio cualitativo — 101 entrevistas entre directivos, profesores y alumnos — realizado en cuatro familias profesionales con alta demanda de formación en Argentina: electricidad, construcción, agroindustria y mecánica automotor. Se propone una mirada que permita recuperar el lugar que la FP ha tenido en diversas fases de los modelos de desarrollo económico colocando el énfasis sobre la formación profesional desde las instituciones educativas para problematizar desde allí diversas trayectorias identificadas que surgen de la intersección en estos campos. En términos teóricos el artículo busca tensionar aquellas interpretaciones que sobredimensionan las lógicas productivas sobre las educativas al momento de diseñar los contenidos de la FP.
\end{abstract}

\section{Palabras clave}

Formación profesional, educación, trabajo, Argentina.

\section{Abstract}

This article seeks to characterize «vocational training» in Argentina. We will analyze the impact of training on workers' carrer path. To this, we carried out a qualitative study in four «professional families» with high demand of training: electricity, construction, agrobusiness and mechanics. We propose to regain the relevance of profetional training in different models of economics developement, emphazing the professional training as an important part of the educational system. The article seeks to reflect on the existing tensions between «productive logic» and «educational logic» that impact on the developement of vocational training contents.

\section{Keywords}

Vocational training, education, labour, Argentina.

\section{Cómo citar/Citation}

Fabian Delfini, Marcelo; Drolas, María Ana y Montes Cató, Juan Sebastian (2021). La formación profesional en la construcción de trayectorias laborales. Estudio sobre sectores productivos en Argentina. Revista de Sociología de la Educación-RASE, 14 (2), 190-204. http://dx.doi. org/10.7203/RASE.14.2.17413.

\footnotetext{
1 El presente artículo se inscribe en el proyecto «Estudio cualitativo sobre egresados de Formación Profesional de sectores electricidad, construcciones, agroindustria y mecánica automotor» Financiado a través del Fondo Nacional de Investigaciones de la Educación Técnico Profesional (FONIETP) perteneciente al Instituto Nacional de Educación Tecnológica (INET) de la Argentina.

2 Marcelo Fabian Delfini, Universidad Nacional de General Sarmiento, mdelfini@campus.edu.ar; María Ana Drolas, Consejo Nacional de Investigaciones Científicas y Técnicas_CONICET_, anadrolas@gmail.com; Juan Sebastian Montes Cató, Centro de Estudios e Investigaciones Laborales —CEIL_, jmontes@ceil-conicet.gov.ar.
} 


\section{Introducción}

En la difícil dialéctica entre educación y trabajo, la opción analítica que significa el abordaje de las trayectorias laborales constituye una alternativa que nos permite visualizar los impactos de los procesos formativos sobre los recorridos profesionales o laborales, además de darnos la posibilidad de observar la pertinencia y los sesgos de las currículas. A partir de esto, poder pensar la interrelación entre los itinerarios de formación y las trayectorias laborales teniendo en cuenta que los primeros instituyen saberes para su instrumentalización en los espacios laborales en tanto saberes de acción. De esta manera pensar que su efectividad en términos de aprendizaje se «mide» a partir de las prácticas y acciones de los sujetos, a partir de los recorridos laborales.

El objetivo principal de este artículo es profundizar la mirada sobre los impactos, en los procesos de inserción en el mundo laboral, de quienes han acreditado un curso de Formación Profesional (en adelante FP) en el sistema educativo, contemplando tanto la mejora en sus condiciones de trabajo como la habilitación para acceder a nuevos empleos, o bien la capacidad de potenciar emprendimientos individuales o colectivos. Esto no significa no poner en juego otras dimensiones tanto micro como macro que también impactan sobre las trayectorias laborales (condiciones del mercado de trabajo, capital social y cultural, género, formación académica, etc.) pero el foco estará puesto especialmente en los procesos formativos.

La investigación estuvo orientada a comprender los impactos institucionales de Formación Profesional, en especial a partir del reconocimiento de las trayectorias de los egresados de las familias profesionales de electricidad, construcción, agroindustria y mecánica automotor a escala nacional. Para ello se recurrió a una estrategia metodológica de carácter cualitativo, a partir de la cual se desarrollaron entrevistas con directores de los centros FP y egresados de los diferentes cursos vinculados a las actividades de referencia.

El artículo se encuentra dividido de la siguiente manera: en principio se desarrolla la metodología seleccionada acorde a los objetivos propuestos en la investigación. En el segundo apartado se presentan las referencias teórico-analíticas desde las cuales se desarrolló el estudio y la interpretación de los hallazgos. En la siguiente sección (Caracterización de la Formación Profesional en Argentina) ubicamos en primer termino a partir de datos cuantitativos la distribución a escala nacional; para luego presentar los datos cualitativos que permiten abordar cuatro posibles tipo de trayectorias en relación a la vinculación entre formación y trabajo. Finalmente, recuperamos en las conclusiones las principales claves interpretativas y posibles preguntas para seguir profundizando sobre la temática.

\section{Metodología y características de la muestra}

El trabajo de campo fue llevado adelante entre agosto y diciembre de 2018 por medio de una estrategia cualitativa de abordaje, con la utilización de diferentes técnicas y herramientas de recolección de la información tendientes a reconocer procesos y hechos significativos para posteriormente describirlos e interpretarlos. La muestra estuvo constituida por 30 Centros de Formación Profesional (CFP) de todo el país, priorizando una distribución equitativa entre las cinco regiones del país: Centro, Noroeste Argentino (NOA) Noreste Argentino (NEA) Cuyo y Sur. En estas se realizaron 71 entrevistas en pro- 
fundidad a egresados de los cursos de FP de las familias profesionales de electricidad, construcción, agroindustria y mecánica del automotor ${ }^{3}$.

El total de entrevistas realizadas fueron 101, de las cuales 30 pertenecen a los directores de las instituciones visitadas y 71 corresponden a egresados. Al respecto, de las 71 entrevistas a egresados, $16 \%$ corresponden a egresados de agroindustria, $15 \%$ a automotores, $38 \%$ a construcción y el resto a egresados pertenecientes a electricidad.

Tabla I. Entrevistas realizadas a directores y egresados por actividad y región

\begin{tabular}{cccccccc}
\hline & CENTRO & CUYO & NEA & NOA & SUR & TOTAL \\
\hline \multirow{3}{*}{ Entrevistas } & Directores & 7 & 6 & 6 & 6 & 5 & 30 \\
\cline { 2 - 8 } & Entrevistas Egresados & 18 & 13 & 12 & 17 & 11 & 71 \\
\cline { 2 - 8 } & Totales & 25 & 19 & 18 & 23 & 16 & 101 \\
\hline
\end{tabular}

Fuente: elaboración propia en base a datos del trabajo de campo.

Del conjunto de las entrevistas realizadas se puede destacar por un lado que el $18 \%$ eran mujeres y por el otro que el $18 \%$ tiene hasta 24 años, $20 \%$ tiene entre 25 y 35, $29 \%$ entre 36 y 45 y $33 \%$ tiene una edad superior a 46 años. En tanto al observar el máximo nivel de estudios alcanzados, 44\% no había terminado la secundaria. En torno a la incorporación de estos en el mercado de trabajo, un 74\% se encontraba ocupado.

El análisis de las entrevistas, se desarrolló en tres partes para facilitar y hacer inteligible la lectura, a partir de los cual generó un sistema de categorizaciones para evaluar las trayectorias en función de: a) las instancias previas a los cursos; b) el tránsito por los CFP y c) las trayectorias posteriores a ese tránsito.

$\mathrm{Al}$ respecto, para el desarrollo analítico se tuvieron en cuenta, dos grandes dimensiones: La primera fue la laboral y la segunda la educativa, cada una ellas atravesó los diferentes momentos de las trayectorias de vida de los estudiantes.

Por su parte para contextualizar el contenido cualitativo de las entrevistas apelamos a datos cuantitativos secundarios del INET que permitieron dimensionar el alcance de esta modalidad en Argentina y de ese modo colocar en contexto los datos obtenidos.

\section{Formación profesional en el marco de la articulación educación y trabajo}

La formación profesional trasciende ampliamente el campo estrictamente formativo y se integra claramente en la intersección de los campos problemáticos de la educación y el trabajo, que es pensada habitualmente como un paso por la capacitación que permitiría generar las condiciones que las miradas desde el mercado de trabajo llaman de «empleabilidad» ${ }^{4}$ Y es justamente en esta última idea donde aparece una de las especificidades de la FP: el joven/adulto como sujeto de política pública educativa. En el campo de

3 La muestra fue suministrada por el INET. Fue construida respetando la distribución en la cantidad de CFP (Centros de Formación Profesional) de cada provincia. $\mathrm{Al}$ respecto, cabe aclarar que dentro de los que denominamos CFP se incluyen por ejemplo los Centros de capacitación para el trabajo (CCT) o los Centros de educación agrario (CEA), diferentes formas de nominación que dependen también de las Jurisdicciones correspondientes. Las entrevistas fueron realizadas en los CFP. A su vez, los datos fueron procesados a partir del paquete estadístico Atlas-ti. En este artículo interesa recuperar el vínculo entre educación y trabajo a través de tópico Formación Profesional que permitirá pensar trayectorias diversas a partir del marco teórico de referencia.

4 El concepto de empleabiliad surge en nuestro país en los años 90 como reflejo de una tendencia mundial en un contexto de fuerte desarticulación de mercado de trabajo y altas tasas de desocupación que dieron nacimiento a múltiples políticas públicas que tendían a buscarle soluciones educativas a los problemas del empleo. Al vincular empleabilidad con desocupación, los problemas de falta de trabajo se trasladan al individuo ocultando los problemas del mercado de trabajo. 
la educación de adultos (llamamos aquí adultos a aquellos que han superado la edad escolar), el trabajo necesariamente ocupa un lugar preponderante, no solo como una manera de contribuir a su formación específica en tanto trabajadores, sino también como parte integrante del acervo con el que las personas adultas se incluyen en las actividades de formación. No es novedoso que buena parte de las definiciones sobre el sujeto adulto, el trabajo, el aprendizaje y la experiencia se hallan en debate al amparo de las tensiones que atraviesan las definiciones de adulto y de trabajo en un ordenamiento social que se transforma.

Si el adulto destinatario de las políticas educativas del siglo xx era varón, trabajador activo, jefe de familia y con experiencia social de independencia, el adulto al cual hoy nos referimos es hombre o mujer, con o sin experiencia de trabajo (más o menos formalizada y protegida, errática en sus trayectorias), con o sin terminalidad educativa anterior, heterogéneo en su edad y con mayores posibilidades de no haber podido aún resolver su independencia económica o habitacional.

En lo que respecta al mundo del trabajo, y sin pretender asumir posiciones rupturistas que exacerban la novedad como muestra del advenimiento de un nueva realidad laboral, las transformaciones estructurales que se sucedieron en nuestro país en décadas pasadas y reeditadas en los últimos años de la década del 10 de este siglo, así como la modificación de las regulaciones que rigen el funcionamiento del mercado de trabajo ha vuelto la experiencia de trabajo visiblemente más heterogénea (anonimizado, 2008) e incierta fundando nuevas formas de subordinación y una estructura productiva segmentada.

Podemos considerar que FP ha sido pensada como un modo más preciso y directo de articulación entre el mundo de la educación/formación y el mundo del trabajo. En su fundamentación y, sin eufemismos, su intencionalidad es la profesionalización de los saberes de oficio y la puesta en valor de la dimensión cognitiva del trabajo ${ }^{5}$. Pero en la actualidad, las instituciones que forman para el trabajo, lo hacen en contextos de inestabilidad del aparato productivo, de cambios en los procesos de trabajo, de demandas cambiantes en relación a las calificaciones/saberes/competencias requeridas y en nuevos marcos discursivos en relación a las formas organizativas de las relaciones laborales, además de haber sido puestas en el centro de las políticas públicas de empleo, como si el desempleo o la precarización e informalidad del trabajo fueran consecuencias de la ausencia o escasez de formación específica de la fuerza de trabajo y responsabilidad del trabajador.

El mercado de trabajo es fuertemente dependiente de las intervenciones públicas. Es el Estado el que regula el intercambio de la fuerza de trabajo; establece quiénes pueden participar y quiénes no; modifica las necesidades de reproducción de la fuerza de trabajo por medio de las políticas sociales; etc. Además, el mercado de trabajo posee una característica específica que es la de no ser homogéneo nunca (no lo fue en los 30 años gloriosos del capitalismo floreciente y no lo es ahora, menos en las regiones marginales del planeta). Existen múltiples mercados de trabajo condicionados por la rama y el sector de la economía, por la zona y las condiciones de acceso, permanencia y movilidad en su interior. Por otro lado, se trata de un espacio segmentado jerárquicamente. Esto significa que no todos pueden acceder a todos los niveles y cada uno de ellos define condiciones específicas para su ingreso, permanencia y promoción.

La relación siempre compleja entre educación y trabajo muestra procesos de acercamiento y distanciamiento y marcos referenciales que están vinculados a los entornos donde se desarrollan. Así, FP

5 Más allá de que FP pudo haberse convertido en los años críticos del neoliberalismo, en una política social de contención, lo cierto es que su génesis puede ser identificada en las necesidades de fuerza de trabajo calificada o semi-calificada. Jamás fue pensada, en sus inicios, como una manera de paliar los problemas del mercado de trabajo. 
en Argentina, construida para la certificación de saberes desarrollados en ese medio, comprende una complejidad mucho más profunda que la relación entre educación y trabajo en la medida que se dirige a jóvenes y adultos insertos en procesos sociales vinculados a la comunidad donde se encuentran. En este sentido, la trayectoria de los sujetos que pasan por ese espacio posibilita entender mejor en qué medida los itinerarios y trayectorias construidas en contextos determinados posibilita la concreción de un trabajo efectivo. En relación a ello, no es sólo la trayectoria laboral previa, su pasaje por el CFP y su trayectoria laboral posterior la que define su inserción, sino que es ello y un contexto estructural que la condiciona o la posibilita además de sus vinculaciones que determinan el capital cultural y social que intervienen en los procesos de inserción laboral.

A diferencia del análisis de las trayectorias desde el mundo del trabajo, pararnos y mirarlas desde el sistema educativo implica reconocer su lógica de largo plazo. Pero este reconocimiento no nos inhibe del diálogo con la inmediatez del mundo productivo en la medida en que la misión de FP es justamente, como dijimos, profesionalizar las actividades y los oficios, creando reglas de acción además de reconocer y valorar su utilidad social. En este sentido FP es una de las maneras que tiene el sistema educativo de lograr articular educación y trabajo en la construcción de las trayectorias de profesionalización de hombres y mujeres con diversos niveles educativos y diferentes experiencias de trabajo y de vida. La pregunta que el sistema educativo se hace (o debiera hacerse en relación a FP y a las trayectorias laborales posibles), a diferencia de la mirada que se puede tener exclusivamente desde el mundo del trabajo, es: ¿qué itinerarios para qué trayectorias? Esto es, qué se aprende y enseña y cómo y dónde el producto de la dialéctica enseñanza/aprendizaje se convierte en un recurso para la acción, se despliega como práctica del sujeto, se instrumentaliza en tanto praxis?

En contextos de desarticulación de los espacios productivos y de incertidumbre de las diversas experiencias de trabajo, ¿cuál es la función que FP puede cumplir? La formación para el trabajo, específicamente la FP, no puede resolver el desempleo, ni la precarización ni la informalidad creciente. Tampoco puede evitar que los trabajadores pierdan sus trabajos. Esto no significa que, a pesar de que no exista una relación lineal entre educación/formación y empleo/desarrollo económico, las políticas de formación para el trabajo constituyan entelequias sociales en la medida en que cualquier proceso formativo (ya sea general o profesional) es siempre positivo. No hay dudas de que nadie sale intocado de las aulas; tanto a nivel de contenidos como en lo que tiene que ver con las vinculaciones y construcción de capital social. Lo que sí debería ser puesto en discusión es la capacidad de los procesos formativos de revertir tendencias ascendentes del desempleo, la precarización y la informalización del empleo, teniendo en cuenta que las causas de estos fenómenos no es la desigual distribución de calificaciones y saberes ni las falencias del sistema educativo y formativo, sino la forma en que se estructuran y regulan los mercados de trabajo en función de los modelos de crecimiento y de distribución del ingreso.

Si FP se piensa como nexo facilitador del ingreso al mercado de trabajo de jóvenes y la reinserción de los adultos que han perdido sus empleos, la pregunta necesaria es ¿para qué trabajo forma FP? Es en este sentido que resulta imposible desvincular los procesos de formación para el trabajo de los modelos de subordinación del empleo y de las formas de la estructura productiva, en la medida en que no es la educación por sí misma la que amplía las oportunidades de ingreso y permanencia en el mercado de trabajo en empleos genuinos y de calidad. 
En Argentina antes de que el Estado notara la necesidad de formación en saberes del trabajo, la formación específica de los obreros era considerada responsabilidad de la industria y los procesos de trabajo que la componen. En los años veinte se crea un dispositivo llamado «aprendizaje», inspirado de alguna manera en el modelo maestro-aprendiz que involucraba la formación específica en el trabajo y estaba destinada a los jóvenes y el desarrollo de los diferentes oficios al interior de procesos productivos diversos. Sobre este modelo es que FP se desarrolló hacia inicios de los años cuarenta.

Fueron los cambios sustantivos en el mercado del trabajo, en las relaciones laborales y en los procesos de trabajo y de distribución del ingreso de las décadas de los 40 y 50 del siglo pasado, los que terminaron de formatear y proyectar FP como una institución necesaria para el desarrollo productivo y de la fuerza de trabajo. De manera conexa y paralela, los sindicatos se consolidan como interlocutores sociales válidos y comienzan a producirse, en base a reivindicaciones sectoriales y luchas gremiales, mejoras en las condiciones salariales y de vida de los trabajadores.

El proceso de ampliación del entramado productivo y creciente poder del sector trabajo en la negociación de su salario (poder basado, entre otras cosas, en la conciencia de ser portadores de saberes y calificaciones específicas y necesarias) fue abruptamente interrumpido en marzo de $1976^{6}$ con la desarticulación total de los marcos protectorios del trabajo. En este contexto, si bien FP dejó de formar parte de la agenda política, continuó creciendo informalmente por la demanda social de los sectores populares que buscaban una formación que les permitiera ingresar al mercado de trabajo o desempeñarse por cuenta propia en algún oficio. Fundadas sobre el legado de la desarticulación de lo que se llamó Estado de Bienestar Periférico, las reformas neoliberales de los 90 lograrán imponer un discurso modernizador como eufemismo con el que se llevó adelante un ataque directo a la estructura productiva industrialista y a la educación técnica en particular. El acento comenzó a ponerse en la educación secundaria general y en los saberes y competencias «requeridos» por el mundo laboral y social en permanente cambio.

En este contexto y en medio de una crisis de empleo inédita y sostenida por los cambios estructurales llevados adelante, se instaló con fuerza la idea de que los problemas de empleo tienen soluciones educativas. Como si la destrucción sistemática del empleo formal se debiera a la baja calificación de la fuerza de trabajo y no a los procesos de globalización y relocalización de las industrias. Así, conceptos tales como empleabilidad y competencias, aparecen para explicar y encarar situaciones problemáticas de vulnerabilidad social a través de FP, que deviene en campo de batalla de diversos intereses.

Estos vaivenes en la estructura económica dados por la itinerancia de las políticas públicas de distribución del ingreso, de regulación del mercado de trabajo y de intervención en la economía, se dieron paralelamente al debate acerca de si en FP debe predominar una lógica educativa o una productiva. La primera implica un rol central de las instituciones educativas en la provisión de la oferta, en el armado de las currículas, en la extensión de certificados que acreditan saberes y, eventualmente, habilitan la continuidad en niveles educativos superiores. Por su parte la lógica productiva de FP asume que ésta última debe responder a las necesidades de calificaciones y formación del entramado productivo, más allá de la efectividad de sus prácticas (en la medida en que no existe itinerario formativo que garantice el acceso al empleo). En esta lógica, las certificaciones aparecen más adaptadas a las necesidades de la demanda de un mercado de trabajo que fluctúa permanentemente y «obliga» a los trabajadores a adaptarse a esas fluctua-

6 Se produce en esta fecha (1976-1983) el golpe cívico-militar que supuso el inicio de una fuerte ofensiva sobre los derechos laborales y las instituciones educativas. 
ciones e incertidumbres.En esta concepción FP se asocia a la noción de «empleabilidad» en cuanto se parte de la idea de que el mercado laboral asume cambios y los trabajadores deben adecuar sus competencias. Precisamente, la noción de competencia, en esta concepción, reemplaza paulatinamente al de calificación.

Si FP fue pensada, en la segunda ola de Industrialización por Sustitución de Importaciones (ISI) ${ }^{7}$ durante las décadas del 40 y 50 especialmente, como una manera de articular las necesidades de una economía en expansión (de empleo y de consumo) y de casi pleno empleo; a partir de la década de los 90 del siglo pasado, con acento en la desregulación general de la economía y una adscripción exacerbada a los principios del neoliberalismo, FP comenzó a ser mirada (mirada que hoy en día parece ser también la hegemónica) como un reservorio de políticas sociales y de contención de los sectores vulnerables en un contexto de destrucción sistemática de fuerzas productivas y de las protecciones y beneficios asociadas al empleo ¿A qué empleo acceden hoy los hombres y mujeres, jóvenes y adultos, que transitan FP? Más adelante en este trabajo, se verá que lejos de insertarse en procesos de trabajo formales, los egresados de FP construyen otras trayectorias diferentes a las llamadas «típicas».

\section{Caracterización de la Formación Profesional en Argentina 4.1. La FP en el marco de su desarrollo a escala nacional}

Interesa a continuación caracterizar FP a partir de los datos cuantitativos recientes ${ }^{8}$. La formación profesional forma parte de la Modalidad Técnico-Profesional. Dentro de esta modalidad la educación de Nivel Secundario es el principal desagregado, con un promedio de 633834 alumnos entre 2012 y 2017 (en torno al 56\% del total). A su vez, es la modalidad que registró mayor incremento en igual periodo, con un aumento del 12,5\%. En segundo lugar, se ubicó FP, modalidad que también acompañó el crecimiento de la enseñanza en Nivel Secundario, registrando un incremento del 11,4\%, pasando de 303976 alumnos en 2012 a 338686 en 2017.

Tomando la distribución geográfica de los alumnos de FP por regiones (NOA, NEA , Centro, Cuyo y Sur), se verifica un patrón de concentración en la zona Centro en desmedro de otras regiones. Más concretamente, la Zona Centro (Entre Ríos, Córdoba, Santa Fe, Buenos Aires y CABA) incrementó su participación en la matrícula en 8,3 puntos porcentuales (pp.), del 55,2\% en 2003 a 63,51\% en 2017.

A nivel jurisdiccional se verifica una concentración aún mayor de la matrícula, dado que la expansión de la zona Centro se explicó esencialmente por el fuerte crecimiento de la demanda en la provincia de Buenos Aires y, en menor medida, el crecimiento en la Ciudad Autónoma de Buenos Aires, siendo las dos jurisdicciones más importantes del país. En conjunto, estas regiones pasaron de concentrar el 45,2\% de la matrícula en 2003 a casi el $60 \%$ en 2017.

El resto de las regiones tuvo dinámicas heterogéneas en el periodo 2003-2017. Esta divergencia entre las trayectorias de las regiones y al interior de cada una de ellas, probablemente se asocie con diversos motivos, como la evolución del mercado de trabajo, las particularidades institucionales de cada lugar, etc. En términos de la desagregación por género, del total de alumnos de FP en el año 2017 (338686), un 43\% eran varones y un 57\% mujeres. Esta preponderancia de las mujeres por sobre los hombres se sostiene en todas las regiones, con una participación relativa similar.

\footnotetext{
Este modelo de desarrollo económico buscaba reemplazar productos industriales con fuerte valor agregado en diversas etapas de complejidad. En una primera instancia a partir de productos con menor inversión hasta llegar a maquinaria pesada.

8 Para esta sección se desarrolló una elaboración propia en base a datos estadísticos de del Ministerio de Educación de la Nación e información provista por INET. 9 Noroeste y Noreste Argentino.
} 
A su vez, si analizamos la distribución de la población en los cursos de Formación Profesional del país por edad, esta tiene una forma asimétrica fuertemente sesgada. Tan sólo en los primeros dos rangos de edad, que abarcan hasta los 24 años, se concentra el 42,5\% de los alumnos. Y si se agrega el siguiente rango, de 25 a 29 años, se supera la mitad de la población, con el 54,3\%. Esta distribución luego tiene un declive progresivo en los sucesivos rangos.

En términos sectoriales, y a nivel regional se observan diferentes patrones de especialización, probablemente asociados a las estructuras productivas y tamaño de mercado local. Por ejemplo, en el Centro se destaca la cantidad de alumnos en Agroindustria (9,5\%), lo cual responde esencialmente a la fuerte demanda en la provincia de Buenos Aires (11,5\%).

Este cuadro de situación a nivel agregado nos permitió observar rasgos salientes en lo referente a la distribución de la población por región, provincia, edad, género y por tipo de familia profesional. Los datos proyectan una semblanza de la población de FP. A continuación interesa abordar los datos cualitativos.

\subsection{Educación y trabajo, articulaciones para pensar la formación profesional}

El trabajo, como se dijo y más allá de los debates abiertos en los últimos 30 años, constituye una actividad central y fundamental en la cual hombres y mujeres no solo obtienen sus medios materiales de vida, sino también construyen formas de estar en el mundo, estar con otros y actuar con reciprocidad. Las maneras en que los trabajadores se posicionan en el mercado de trabajo tienen sus consecuencias relativas en las formas de inserción en el empleo, más allá de que el mercado de trabajo tenga lógicas que escapan a las estrategias individuales de posicionamiento. FP en tanto modalidad educativa tiene como una de sus motivaciones centrales contribuir en ese posicionamiento en la medida en que los jóvenes-adultos que participan de sus programas formativos tienen la motivación laboral como eje de su búsqueda educativa. Y es en este sentido que FP no es una modalidad más, ni una modalidad menor y mucho menos una estrategia para resolver problemas del mercado de trabajo o una política de empleo (aunque pueda acompañar este tipo de políticas) o una institución de contención social. A lo sumo, sí podemos pensarla como herramienta para atenuar las desigualdades de base en relación a los movimientos del mercado de trabajo en la medida en que su impacto más directo y relevante es sobre los sujetos.

En referencia a la muestra con la que se trabajó y que caracterizamos, se observa que las trayectorias laborales que preceden a la realización de los cursos son trayectorias que podemos definir como no lineales. Esto es, trayectorias fluctuantes en trabajos no formales, de corta duración y sucesivos dentro o fuera de la misma o semejante actividad. Como línea general a la tendencia global en cuestiones laborales, las trayectorias en otro tiempo llamadas «típicas» en las que el empleo es por tiempo indeterminado, en blanco y con todos los beneficios que estipula la Ley de Contrato de Trabajo (LCT), son las menos. El escenario general es de informalidad tanto en empleados como en autoempleados y de formalidad (los menos) para muchos de los que realizaron el curso para la utilización de los saberes en sus hogares y que ejercen su profesión en otras actividades o se encuentran retirados o jubilados.

En cuanto al primer grupo, el de las trayectorias zigzagueantes, encontramos realidades tan diversas como unificadoras en tanto experiencias no lineales: la constante inseguridad laboral asumida, por otro lado, como la normalidad dentro de los parámetros del empleo. Ya se trate de actividades vincula- 
das a agroindustria, automotor, electricidad o construcción, el denominador común es la informalidad de los vínculos laborales o del auto-empleo y la sinuosidad de las trayectorias.

La visualización de estas trayectorias laborales anteriores a los cursos es útil para el análisis de las trayectorias posteriores en las que se vieron proyectadas las intencionalidades con las que se realizaron los cursos: aprender un oficio para conseguir empleo, perfeccionarse en nuevas técnicas, obtener un certificado de los conocimientos puestos en práctica todos los días o para adquirir conocimientos para su aplicación privada en los hogares. En cualquiera de los casos y como dijimos, el impacto del proceso educativo es innegable más allá del grado en que pueda darse, porque nadie sale intocado de los procesos de construcción colectiva de saberes.

Más allá de estas consideraciones, y pensando en la relación de los cursos de referencia realizados por los egresados entrevistados y las trayectorias laborales inmediatamente posteriores a la realización de los mismos, podemos pensar algunas categorías descriptivas de los impactos de los itinerarios formativos en estas trayectorias, que si bien no son excluyentes, sí constituyen situaciones que podemos llamar típicas construidas en base a la puesta en relación entre el proceso de aprendizaje particular y su impacto en el empleo.

1. trayectorias 'débiles' en la actividad laboral: este tipo de trayectorias se da con mayor presencia en los cursos pertenecientes a la familia profesional agroindustria. Entre los egresados sobresale la primera intención de aprender para sí mismos sin finalidad comercial o laboral, aunque esta opción aparece como una posibilidad en la medida que la encuentran factible para complementar los ingresos familiares. En el caso específico de apicultura, destaca el hecho de que los egresados de estos cursos concurren con la clara idea de encarar un proceso de aprendizaje para emprendimientos laborales o perfeccionarse/certificarse en la actividad que ya se venía realizando.

«Yo quiero hacer un emprendimiento... ahora por la economía está muy complicada... pero si estoy almacenando conocimiento de todo porque si quiero, quiero tener una empresa. Pero estoy recaudando conocimiento, a mí me sirve». (2-02-002-E-1).

«Porque uno al estar realizando una formación profesional en determinados...(pausa) en determinadas carreras por decirlo de alguna forma ya te da posibilidad, de que vos ya tenés un perfeccionamiento para el día de mañana encarar ese trabajo». (7-17-001-E-1).

La debilidad de este tipo de trayectorias viene dada por la oscilación en la actividad laboral referida al tema específico del curso. Podemos decir que se trata del aprendizaje de saberes que conforman una actividad refugio. Como una especie de reservorio de un saber hacer para situaciones en las que se hace necesario completar los ingresos familiares.

Una cuestión a remarcar y que en el contexto del trabajo realizado adquiere relevancia, es el hecho de que los cursos de la familia profesional de agroindustria los realizan en su mayoría mujeres que concurren para perfeccionarse o aprender y suelen hacer más de un curso de esa familia. La feminización de la matrícula en estos cursos y el hecho de ser contemplados como una fuente potencial de ingresos, no constituyen datos menores teniendo en cuenta los valores 
del desempleo y los ingresos femeninos que suelen ser mayores en el primer caso y menores en el segundo.

2. trayectorias fuertes en la actividad laboral: existen cursos de familias profesionales que podemos caracterizar de esta manera. Hablamos de construcción y electricidad especialmente, que si bien pueden darse formas precarias en las condiciones de trabajo posteriores a la realización de los cursos (con precarias nos referimos a condiciones de informalidad total o cuentapropismo con o sin inscripción en la Agencia Federal de Ingresos Públicos, encargada de la recaudación, en adelante AFIP) constituyen trayectos formativos de alto impacto en aquellos casos en los que no existía experiencia previa en la actividad. La fortaleza de este tipo de trayectorias viene dada por la proyección hacia adelante que hacen los egresados en función del curso realizado. Son capaces de planificar emprendimientos, constituir sociedades con compañeros del curso, comprar herramientas con la idea de asentarse en la profesión, etc. Este tipo de saber hacer es percibido por los egresados como un saber socialmente legitimado y valorado. En este sentido una frase muy escuchada en las entrevistas es «trabajo de lo que estudié en el curso», sea en solitario, con otros o para terceros el hecho de la realización del curso constituyó un punto de inflexión en muchas trayectorias laborales. Esta idea se pone en evidencia en los siguientes tramos.

«Estoy con otra persona, pero ya estamos como socios digamos, yo consigo trabajo él va conmigo, él consigue y yo voy con él y así, y hacemos. Si contratamos a un chico para que nos ayude lo contratamos y le pagamos los dos, las herramientas son a medias. Siempre el trabajo es de electricidad». (1-01-001-E-4).

"Yo ya estoy trabajando en la actualidad, hago trabajos de mecánica mantenimiento de vehiculos nafteros, y pronto a integrarme en lo que es el desarrollo electrónico de los automóvil de hoy, sofisticados, asi que es lo que estoy... actualmente siempre desenvolviéndose en el aprendizaje y el desarrollo de lo que es, este (pausa) la industria automotrià. (1-01-003-E-2).

«Me puse un taller de carpintería en mi casa y hago muebles todo... y habrá sido en el tercer año, primero empecé el curso después bice el taller». (04-09-001-E-3).

Un dato significativo es que los cursos de la familia profesional electricidad se encuentran concatenados y la mayoría de los egresados realizaron todos los cursos o tienen pensado hacerlo en la medida en que ello amplía sus posibilidades de empleo ya que existen muchas tareas específicas que deben ser realizadas por electricistas con matrícula registrada. En esta categoría interpretativa se ve más notoriamente lo que se dijo más arriba acerca de la apropiación de los recursos para la acción que no es otra cosa que la apropiación, en la práctica cotidiana, del saber aprendido.

3. trayectorias con persistencia laboral en la actividad: que nos habla de aquellos que, siendo idóneos, la realización del curso responde a la obtención de la matrícula para la ampliación de su campo laboral o la independización de terceros para trabajar por cuenta propia. Esta situación puede implicar o no la inscripción en la $\operatorname{AFIP}^{10}$ que constituye, más allá de su condición de

10 Administración Federal de Ingresos Públicos. 
precariedad según las referencias que nos hablan de lo que se entiende por condiciones típicas de empleo, cierto rasgo de formalidad/informalidad en las tareas realizadas. Mayormente, la inscripción en la AFIP no está generalizada ni constituye una preocupación entre los egresados. Es relevante el hecho de que esta persistencia en la actividad posterior a la realización del curso, viene dada por la obtención del certificado que acredita formalmente los conocimientos puestos en práctica en la actividad desarrollada de forma cotidiana. Esto es, en este caso, FP funciona como nodo legitimador de una actividad ya sabida o conocida por el egresado. Se trata de un proceso de acreditación de los saberes que el cursante trae al espacio del aula al mismo tiempo que una forma de completarlos. Es en este proceso que FP valoriza y reconoce la utilidad social de los saberes existentes más allá del proceso estricto de enseñanza-aprendizaje.

«Yo ya hace 8 años que trabajo como electricista, no es cierto y... en muchos casos, en la mayoria, siempre me he enfrentado a situaciones en las que me hacia falta una certificación por ejemplo... De hecho [...] perdí trabajos, a veces no quise tomar 87 también trabajos por falta de matrícula, porque algunos planos no me los reconocen porque no tenía el modo de certificar mis conocimientos al respecto. Entonces se me empezó a prender la lucecita, por sugerencia de algunos empleadores mios como arquitectos, como otros ingenieros por abi que para obras sencillas siempre me llevaba para trabajar. Entonces como para que se me facilitara un poco y así ganar un poco más de dinero». (4-08-001-E-4).

«Si bien yo tenía conocimientos de electricidad, fui tomando base sólida de en cuanto a cálculos, en cuanto a medida de seguridad, y bueno aparte hacerlo más técnico aquello que yo ya sabia, si bien tenía experiencia en lo que es eléctrico (...) yo venía de otro rubro y bueno cuando decido implementar mis conocimientos de electricidad en una empresa constructora, entendí que la demanda requería de tener mayor conocimiento y un certificado que avale el conocimiento y el poder abocarlo a la práctica con normativas vigentes, y bueno es eso lo que lo que llevó a hacer los cursos, y bueno fue así, aprendí la parte técnica, que podía hacer una instalación domiciliaria». (1-04-001-E-4).

Otra de las cuestiones centrales que caracteriza estas trayectorias es la necesidad de actualizarse en nuevas técnicas, nuevos materiales y nuevas formas de trabajo en la actividad que ya se está realizando o con la motivación de completar conocimientos complementarios a la actividad laboral central.

"Comencé el tema de la construcción con un amigo, un hombre mayor albañil, me enseñó mucho, siempre laburé con ese hombre, hasta que me independicé y bueno estoy con todos los cursos, fui ampliando las ramas de la construcción, boy en dia prácticamente ya no hago más albañilería. Me fui especializando, dentro de la construcción pero en otras cosas». (06-16-001-E-3). 
4. sin trayectoria laboral posterior en la actividad a la que refiere el curso: existe un grupo de egresados que no han tenido inserción alguna ni en la actividad de la que se trataba el curso realizado ni en relación a ninguna otra actividad. En muchos casos, se trata de personas que asisten al CFP como espacio de socialización o de aprendizaje en un sentido estricto pero cuya dedicación laboral permanente corre por otros carriles. Por ejemplo, jubilados y jubiladas que utilizan su tiempo para estudiar y construir lazos comunitarios y trabajadores en actividades variadas que realizan los cursos con la intención de utilizar esos conocimientos en sus hogares o por gusto personal y muchas veces con la proyección futura de completar ingresos realizando como actividad paralela los conocimientos construidos en los cursos.

«Yo podría vender mermelada o escabeche, qué sé yo, o hacer muchas cosas y vender, ¿̇y qué ganaría? un bolsillo un poco más lleno, yo tengo una buena jubilación, muy buena jubilación porque yo llegué a comisión inspector, que es un cargo más que comisario». (2-06-002-E-1).

«Lo aplico en mi casa, en mi familia y a alguien que le puedo dar una mano». (3-07-002-E-2).

En esta categoría analítica se encuentran también los casos como el que se grafica a continuación y que tiene que ver con el género del egresado. Es el caso de una mujer egresada de un curso de mecánica que quedó sólo en el gusto de hacerlo no porque no exista el deseo de poner en acto lo aprendido, sino porque su condición de género le impone límites a esa posibilidad. Reales o no, el género impone un límite al desarrollo de la actividad laboral relacionada con lo aprendido.

«No estoy trabajando de eso. Porque para una mujer es más difícil meterse en un tallery eso». (13-27-001-E2).

Con el análisis y construcción de estas categorías o formas típicas lo que se quiso poner en relieve es que aun en aquellos casos en que no existe inserción posterior o que ésta es débil, los saberes y conocimientos son percibidos como recursos para la acción. Son puestos en valor no sólo en la práctica cotidiana, sino como reservorio de posibilidades. El paso por FP, con la disposición y con el bagaje de saberes que fueren y aún como contrapartida a un plan social, marca una experiencia que siempre es valorada positivamente por el egresado. No es un dato menor comprobar la transformación de la realidad del sujeto adulto a partir del impacto de los procesos formativos más allá de la trayectoria laboral posterior en relación al curso realizado.

Ahora bien, en ninguna de las categorías construidas en base a las regularidades observadas en el análisis de las entrevitas, podemos ponderar el hecho de que los egresados de los procesos formativos de FP, se hubieran insertado en procesos de trabajo formales. Esto es, empleos bajo relación de dependencia con las cargas y beneficos sociales correspondientes. Ni siquiera podemos hablar de inserción en las formas fraudulentas del trabajo dependiente (EXPLICAR) . Si bien FP abre algunas puertas al mundo del trabajo, estas puertas no suelen ser las del trabajo genuino y de calidad, sino más bien las puertas del cuentapropismo y tareas realizadas en la informalidad que completan ingresos familiares. A partir de acá la pregunta vuelve a ser la misma, 
¿formar para qué trabajos? ¿Qué es primero: ¿la formación o la existencia de un mercado de empleo cuidado, regulado y con un Estado presente en el control de sus excesos?

De más está decir que la formación como estrategia de supervivencia, es una estrategia válida en contextos de alto desempleo y alta informalidad. Válida y necesaria. Pero no es la formación para el trabajo la que va a resolver los problemas de empleo reales ni las cuestiones relativas a la desigual distribución del ingreso. A lo sumo atenúa los problemas de ingreso de quienes sobrellevan los problemas de empleo recurriendo a las estrategias formativas, pero es el Estado a través de sus políticas públicas el que debe generar las condiciones de posibilidad para el empleo seguro y no el trabajador desempleado, formándose.

\section{Conclusiones}

La formación profesional constituye un mundo poco explorado en Argentina. No porque no se haya escrito e investigado sobre el tema ni porque no sea materia de preocupación tanto entre los expertos en políticas públicas educativas como para estudioso en la materia. Es un mundo inexplorado e infravalorado socialmente (es de remarcar que la valoración social de FP depende, como de muchas otras cuestiones, del nivel de institucionalización y formalización por parte de las instituciones del Estado). FP es desde nuestra perspectiva, una de las modalidades más potentes del sistema educativo y, al mismo tiempo, la modalidad con mayores limitaciones y problemas a sortear.

Hemos señalado a partir del trabajo de campo que los procesos educativos en FP deben estar diseñados para construir herramientas de intervención en la realidad y modificar las condiciones y contextos. En función de ello observamos que las trayectorias laborales que preceden a la realización de los cursos son trayectorias no lineales, fluctuantes en trabajos no formales, de corta duración y sucesivas dentro o fuera de la misma o semejante actividad. Resaltamos que el proceso de apropiación de los recursos para la acción va más allá del adquirido en el espacio educativo propiamente dicho. Contemplando este proceso que construye trayectorias identificamos, al adentrarnos en las actividades de electricidad, construcciones, agroindustria y mecánica automotor, cuatro tipo de trayectorias que definimos como trayectorias 'débiles' en la actividad laboral; trayectorias fuertes en la actividad laboral; trayectorias con persistencia laboral en la actividad; y sin trayectoria laboral posterior en la actividad.

A partir de estos hallazgos consideramos que en términos analíticos surgen una serie de ejes que resultan claves para comprender la dinámica actual y posibles desafíos de la FP. En primer lugar, es un instrumento inmejorable para salvar o al menos atenuar la grieta entre trabajo e instrucción (Jiménez, 2009) por lo cual no puede ignorar la realidad del mercado de trabajo ni la realidad del empleo, aunque esto no signifique olvidar su inscripción dentro del sistema educativo y la necesidad de articular didácticas especializadas. Reconocer la importancia de aprender en y para el trabajo no significa renunciar a la mirada específicamente educativa que debe sostener la modalidad. En este sentido, FP no debe confundirse con formación calificante o con formación por competencias. Estas últimas dos corresponden a los ámbitos productivos sectoriales exclusivamente y son tanto las empresas como los sindicatos los encargados de formularlas, llevarlas adelante y certificarlas de la manera que les resulte más conveniente según negociación. FP como institución del sistema educativo tiene otras lógicas internas y externas que le dan especificidad, como ser por ejemplo, su carácter compensador y no reforzador de las desigualdades educativas, sociales y laborales. 
En segundo lugar, teniendo en cuenta el desarrollo del artículo en función del uso de la noción de trayectoria para el análisis del vínculo formación-trabajo en el ámbito de FP, no solo podemos pensar formatos de las trayectorias inmediatamente posteriores, sino también formas típicas de atravesar los procesos formativos de FP: 1) de manera paralela a la actividad laboral (como actualización); 2) anterior al ingreso al mercado de trabajo (como aprendizaje de oficio propiamente dicho) y 3 ) como recurso en situación de desempleo. Este constituye otro de los problemas que FP debe afrontar: hacerse consciente de esta heterogeneidad y actuar en consecuencia preguntándose ¿qué oferta para qué demanda?, ¿qué itinerario ofrecer para cada una de estas realidades? Y este problema es al mismo tiempo una gran potencialidad que tiene la educación de adultos en general y FP en particular: la posibilidad de ofrecer itinerarios diferentes y diferenciados acerca de la misma temática. Problema este que se enlaza con la posibilidad de acreditar saberes y no solo certificarlos.

En tercer lugar, FP tiene el problema de a pesar de ser una modalidad pensada en función de su impacto en la inserción del cursante en el mercado de trabajo, no tiene ni estipula sistemas institucionalizados de seguimiento de egresados para dar cuenta de la efectividad de sus currículas, de sus didácticas específicas y de los formatos de cursada. Como vimos en el apartado anterior, las trayectorias típicas que se plantearon necesitan de una mayor sistematicidad y eso se logra con la construcción de datos más abarcativos y en función de cada una de las familias profesionales. Un sistema de seguimiento de egresados y análisis de datos, articulado con el análisis de las condiciones de inserción, tendría un impacto positivo en la construcción de los trayectos formativos además de brindar herramientas de intervención.

En definitiva, creemos que FP constituye un campo de intervención educativa en el que vale la pena posar nuestras miradas para repensarla como una de las modalidades que necesitan ser tonificadas y no solo en función de a quienes está dirigida (los jóvenes y adultos constituyen un universo tan interesante como difícil), sino especialmente en disputa con ciertos intereses que hacen de FP un campo de disputas que le quitan especificidad, alcance y valor real en tanto espacio de construcción de recursos de y para la acción en un espacio tan problemático como es el espacio laboral, sus relaciones y sus conflictos.

\section{Referencias bibliográficas}

Delfini, Marcelo y Spinosa, Martín (2008). Trabajo Argentino. Cambios y continuidades en 25 años de democracia. Buenos Aires: Biblioteca Nacional-UNGS.

Frassa, Juliana y Muñiz Terra, Leticia (2004): “Trayectorias laborales: origen y desarrollo de un concepto teórico-metodológico”. Cuartas Jornadas de Etnografía y Métodos Cualitativos, IDES, Agosto 2004.

Gallart, María Ana (1998): “Tendencias y Desafíos de la Formación Profesional y el Empleo en América Latina”. La Educación. Revista Interamericana de Desarrollo Educativo. Washington: OEA, XLII-I-III.

Jacinto, Claudia (2015): “Nuevas lógicas en la formación profesional en Argentina. Redefiniendo lo educativo, lo laboral y lo social". Perfiles educativos, 37 (148), 120-137.

Jimenez, Mariela (2009): “Tendencias y hallazgos en los estudios de trayectoria: una opción metodológica para clasificar el desarrollo laboral”. Revista Electrónica de Investigación Educativa, 11 (1), 1-21.

Millenaar, Verónica (2012): “Vínculos con el trabajo e identificaciones de género. La relación con la actividad en el análisis de trayectorias laborales de mujeres jóvenes”. Desarrollo Económico, 25, 73-99. 
Millenaar, Verónica (2014). “Trayectorias de inserción laboral de mujeres jóvenes pobres: el lugar de los programas de Formación Profesional y sus abordajes de género". Revista Trabajo y Sociedad, vol. XVII (22), 325-339.

Spinosa, Martín y Testa, Julio (2009): “L’Enseignement professionnel en Argentine entre volontarisme et isolement dans la recherche d'un pays posible”. Revue Formation et emploi, 107, 9-24.

Verdier, Eric Verdier (2008). "L'éducation et la formation tout au long de la vie: une orientation européenne, des régimes d'action publique et des modèles nationaux en évolution”. Sociologie et Sociétés, $40(1), 195-225$.

\section{Notas biográficas}

Marcelo Fabian Delfini es investigador independiente del Consejo Nacional de Investigaciones Científicas y Técnicas —CONICET/Universidad Nacional de General Sarmiento (UNGS)—. A su vez se desempeña como Docente Universidad de Buenos Aires — UBA — y de la Universidad Nacional de Rafaela —UNRaf - en Argentina. Es Doctor en Ciencias Sociales por la UBA y Licenciado en Sociología por la misma universidad. Desarrolló estancias de investigación en la Universidad Libre de Bruselas. Investiga en áreas de sociología del trabajo; trabajo y educación, con particular interés por los procesos de formación profesional. Posee publicaciones vinculadas a las relaciones del trabajo en Argentina, sindicalismo, educación y trabajo.

María Ana Drolas es investigadora Adjunta del Consejo Nacional de Investigaciones Científicas y Técnicas —CONICET — y se desempeña en Centro de Estudios e Investigaciones Laborales —CEIL-. Es Doctora en Ciencias Sociales por la Universidad de Buenos Aires —UBA—, posee Master por la Universidad Complutense de Madrid — UCM — y Licenciada en Ciencias Políticas por la UBA. Ha desempeñado actividades de docencia de posgrado y participado en congresos orientados a problematizar los vínculos entre educación y trabajo. Sus áreas de investigación son la sociología del trabajo y los vínculos entre educación y trabajo colocando el énfasis a la formación profesional. Posee publicaciones en el campo de la educación y trabajo y en sociología del trabajo.

Juan Sebastian Montes Cató es investigador principal en el Centro de Estudios e Investigaciones Laborales —CEIL_ del Consejo Nacional de Investigaciones Científicas y Técnicas —CONICET_- Se desempeña también como profesor de la UBA en el nivel de grado y posgrado y en la Universidad Nacional de Moreno - UNM- Es Doctor en Ciencias Sociales; Magíster en Ciencias Sociales del Trabajo y Licenciado en Sociología por la Universidad de Buenos Aires. Desarrolló estancias de docencia e investigación en España, Francia, Bélgica y Brasil. Investiga en áreas de sociología del trabajo, relaciones laborales y sociología de la educación (en particular la relación entre trabajo y formación). Posee publicaciones vinculadas a las relaciones del trabajo, sindicalismo, educación y trabajo. 